\title{
Magnonic Holographic Imaging of Magnetic Microstructures
}

\author{
D. Gutierrez ${ }^{1}$, H. Chiang ${ }^{1}$, T. Bhowmick ${ }^{1}$, A.D. Volodchenkov ${ }^{1}$, M. Ranjbar ${ }^{1}$, G. Liu ${ }^{1}$, C. Jiang ${ }^{1}$, \\ C. Warren', Y. Khivintsev ${ }^{2,3}$, Y. Filimonov ${ }^{2,3}$, J. Garay ${ }^{1}$, R. Lake ${ }^{1}$, A.A. Balandin', and A. Khitun ${ }^{1}$ \\ ${ }^{1}$ Department of Electrical and Computer Engineering, University of California - Riverside, \\ Riverside, California, USA 92521 \\ ${ }^{2}$ Kotelnikov Institute of Radioengineering and Electronics of Russian Academy of Sciences, \\ Saratov Branch, Saratov, Russia 410019 \\ ${ }^{3}$ Saratov State University, Saratov, Russia 410012
}

\begin{abstract}
We propose and demonstrate a technique for magnetic microstructure imaging via their interaction with propagating spin waves. In this approach, the object of interest is placed on top of a magnetic testbed made of material with low spin wave damping. There are micro-antennas incorporated in the testbed. Two of these antennas are used for spin wave excitation while another one is used for the detecting of inductive voltage produced by the interfering spin waves. The measurements are repeated for different phase differences between the spin wave generating antennas which is equivalent to changing the angle of illumination. The collected data appear as a 3D plot - the holographic image of the object. We present experimental data showing magnonic holographic images of a low-coercivity Si/Co sample, a high-coercivity sample made of $\mathrm{SrFe}_{12} \mathrm{O}_{19}$ and a diamagnetic copper sample. We also present images of the three samples consisting of a different amount of $\mathrm{SrFe}_{12} \mathrm{O}_{19}$ powder. The imaging was accomplished on a $\mathrm{Y}_{3} \mathrm{Fe}_{2}\left(\mathrm{FeO}_{4}\right)_{3}$ testbed at room temperature. The obtained data reveal the unique magnonic signatures of the objects. Experimental data is complemented by the results of numerical modeling, which qualitatively explain the characteristic features of the images. Potentially, magnonic holographic imaging may complement existing techniques and be utilized for non-destructive in-situ magnetic object characterization. The fundamental physical limits of this approach are also discussed.
\end{abstract}

(C) 2016. This manuscript version is made available under the Elsevier user license http://www.elsevier.com/open-access/userlicense/1.0/ 


\section{Introduction}

Magnetic micro and nanostructures have found a wide range of practical applications starting from data storage [1] to biotechnology and medicine [2]. The understanding of magnetic dynamics is the key for building more efficient devices (e.g. more scalable and less power consuming magnetic memory [3], accurate and robust magnetic field sensors [4], customized magnetic nanoparticles for drug delivery [5]). There are different techniques for magnetic microstructures characterization and imaging including magnetic force microscopy [6], magneto-optic Kerr rotation [7], X-ray [8] and electron diffraction [9]. Each of these techniques possesses its own advantages and limitations. For instance, magnetic force microscopy provides a nanometer spatial resolution but has relatively short lateral scanning [10]. Optical methods make it possible to study ultra-fast spin dynamics with picosecond time resolution [11]. The most advanced near-field Brillouin light scattering technique, makes it possible to map spin wave modes with the spatial resolution of few tens of nanometers [12]. There is a great impetus in the development of novel techniques for magnetic materials imaging and characterization. In this work, we describe a method of imaging magnetic microstructures via their interaction with propagating spin waves.

Spin wave is a collective oscillation of spins in a spin lattice around the direction of magnetization. Spin waves appear in magnetically ordered structures, and a quantum of spin wave is called a "magnon". The collective nature of spin wave phenomena manifests itself in relatively long coherence length, which may be order of the tens of micrometers in conducting ferromagnetic materials (e.g. $\mathrm{Ni}_{81} \mathrm{Fe}_{19}$ ) and exceed millimeters in non-conducting ferrites (e.g. $\left.\mathrm{Y}_{3} \mathrm{Fe}_{2}\left(\mathrm{FeO}_{4}\right)_{3}\right)$ at room temperature [13]. The latter makes it possible to build magnonic interferometers exploiting spin waves within the coherence length. For example , a Mach-Zehnder-type spin wave interferometer based on YIG structure was demonstrated in 2005 by M. Kostylev et al. [14]. The phase difference among the interfering spin waves was controlled by the magnetic field produced by an electric current flowing through a conducting wire under the one of the

arms. In general, spin wave dispersion depends on the strength and direction of the bias magnetic field [15]. Even relatively weak (e.g. tens of Oersted) magnetic field produced by a micro-scale magnet placed on the top of magnonic waveguide may result in a prominent phase shift. This phenomenon is used in Magnonic Holographic Memory [16]. In this approach, the read-out of magnetic memory elements is accomplished by measuring the inductive voltage produced by the interfering spin waves propagating through the matrix consisting of magnetic waveguides with magnetic memory elements placed on top. Experimental data obtained for the first working prototypes have demonstrated a great potential of using spin wave interference for parallel magnetic bit read-out, where different configurations of magnets in the matrix result in different output inductive voltage $[16,17]$. Here, we extend this approach to magnetic microstructures imaging. The rest of the paper is organized as follows. In the next Section II, we describe the principle of magnonic holographic imaging. In Section III, we describe experimental setup and present experimental data: holographic images of different magnetic microstructures. Experimental data are 
complemented by the results of numerical modeling. The Discussion and Conclusions are given in Sections IV and V, respectively.

\section{Principle of Operation}

The idea of magnonic holographic imaging is illustrated in Fig.1. The testbed is a cross junction made of material with low spin wave damping. It is a four-terminal device with four micro-antennas fabricated at the edges of the cross. Two of these antennas (e.g. \#1 and \#3 as depicted in Fig.1) are used for spin wave generation, while the one of the other antennas (e.g. \#2 or \#4) is used for detecting the inductive voltage produced by the interfering spin waves. There is a microwave system which includes phase shifters and attenuators to control independently the amplitudes and the phases of the input spin waves. The object of interest is placed on the top of the junction. It may be a micro-magnet, magnetic powder, or an ensemble of magnetic nanoparticles. Magnetic field produced by the object affects the propagation of spin waves through the junction and changes the output inductive voltage. The measurements are repeated for the different combinations of the input phases, where the phase at each input port is independently varied from 0 to $2 \pi$. As a result, one obtains a collection of data (Phase1, Phase 2, Inductive Voltage). Plotting the inducting voltage as a function of the input phases, we obtain a 3D image of the object in the phase-voltage space.

The process of obtaining holographic image can be modeled by using the Landau-Lifshitz-Gilbert (LLG) equation $[18,19]$ :

$$
\frac{d \vec{m}}{d t}=-\frac{\gamma}{1+\alpha^{2}} \vec{m} \times\left[\vec{H}_{e f f}+\alpha \vec{m} \times \vec{H}_{e f f}\right],
$$

where $\vec{m}=\vec{M} / M_{s}$ is the unit magnetization vector, $M_{s}$ is the saturation magnetization, $\gamma$ is the gyromagnetic ratio, and $\alpha$ is the phenomenological Gilbert damping coefficient. The first term of equation (1) describes the precession of magnetization about the effective field and the second term describes its relaxation towards the direction of the field. The effective field is given as follows:

$$
\vec{H}_{e f f}=\vec{H}_{d}+\vec{H}_{e x}+\vec{H}_{a}+\vec{H}_{b}
$$

where $H_{d}$ is the magnetostatic field, $H_{e x}$ is the exchange field, $H_{a}$ is the anisotropy field, and $H_{b}$ is the external magnetic field. In general, LLG equation allows us to simulate all operational steps including spin wave generation by the $A C$ magnetic field produced by the input antennas, spin wave propagation through the cross junction, interaction with the object of interest, and find magnetization at the output terminal as a result of spin wave interference. However, the direct solving of LLG equation for micrometer scale structures is quite a challenging task requiring a lot of computer resources. The need of simulating spin wave transport with different input phases further increases the computational time.

In order to simplify the calculation procedure, we introduce an empirical model based on the known solution of the LLG equation and measured transport characteristics. Spin wave $\vec{m}_{i}(r, t)$ generated by 
the $i$-th input antenna can be described as follows:

$$
m_{i}(r, t)=A_{i} \cdot \exp [-\kappa r] \cdot \sin \left(k r-\omega t+\phi_{i}\right),
$$

where the dynamic part can described as a propagating wave (magnetization wave), $A_{i}$ and $\phi_{i}$ are the initial amplitude and the phase of the spin wave, $k$ is the wave vectors, $\kappa$ is the damping constant, $r$ is the distance travelled, $\omega$ is the frequency. It should be noted that the different types of spin waves possess significant different dispersion $k(\omega)$. For instance, magnetostatic spin waves propagating parallel to the bias magnetic field (so-called backward volume magnetostatic spin waves BVMSW) possess negative group velocity $v_{g}=\partial \omega / \partial k<0$, while spin waves propagating perpendicular to the bias magnetic field (so-called magnetostatic surface spin waves MSSW) possess positive group velocity $v_{g}>0$ ) [15]. Next, we introduce empirical relations between the spin wave amplitude $A_{i j}$ and phase $\phi_{i j}$ generated at the $j$-th output port by the spin wave generated at the $i$-th input $A_{j}, \phi_{j}$ as follows:

$$
\begin{aligned}
& A_{i j}=\sigma_{\mathrm{ij}} \times A_{i}, \\
& \phi_{i j}=\phi_{i}+\Delta_{\mathrm{ij}},
\end{aligned}
$$

where $\sigma_{\mathrm{ij}}$ is the amplitude change, $\Delta_{\mathrm{ij}}$ is the phase shift. Wave vector $k_{0}$, operational frequency $\omega$, and damping constant $\kappa$, are defined by the antennas geometry, spin wave generator, and material properties of the testbed material, respectively. The amplitude change $\sigma_{\mathrm{ij}}$ and the phase shift $\Delta_{\mathrm{ij}}$ are extracted from the experimental data. The magnetization change at the $j$-th output is a sum of the two interfering spin waves:

$m_{j}(r, t)=\sum_{i} A_{i j} \cdot \exp \left[-\kappa r_{i j}\right] \cdot \sin \left(k r_{i j}-\omega t+\phi_{i j}\right)$.

Finally, the output inductive voltage is found according to the Faraday's law, the magnitude of the inductive voltage is proportional to the speed of the magnetic flux change $V_{\text {ind }}=-d \Phi_{m} / d t$. It can be calculated as follows [20]:

$V_{\text {ind }}=\frac{\mu_{0}}{2} \int \vec{H} \cdot \frac{d \vec{m}(r, t)}{d t} d \vec{r}$

where $\mu_{0}$ is the magnetic constant, $H$ is the magnetic field, the integration is over the output antenna contour. The inductive voltage oscillates with the same frequency $\omega$ as the propagating spin waves. The amplitude of the inductive voltage has maxima when spin waves are coming in phase (constructive interference). The output has minima if the spin waves are coming out of phase (destructive interference).

\section{Experimental Setup and Holographic Imaging}

The schematics of the experimental setup are shown in Fig. 2(A). The testbed is a cross junction made of single crystal yttrium iron garnet $\mathrm{Y}_{3} \mathrm{Fe}_{2}\left(\mathrm{FeO}_{4}\right)_{3}(\mathrm{YIG})$ film epitaxially grown on top of a Gadolinium 
Gallium Garnett $\left(\mathrm{Gd}_{3} \mathrm{Ga}_{5} \mathrm{O}_{12}\right)$ substrate using the liquid-phase transition process. After the films were grown, micro-patterning was performed by laser ablation using a pulsed infrared laser $(\lambda \approx 1.03 \mu \mathrm{m})$, with a pulse duration of $\sim 256 \mathrm{~ns}$ and average power of $20 \mathrm{~W}$. The radius of the laser spot is about $50 \mu \mathrm{m}$, the speed of scanning is $48 \mathrm{~cm} / \mathrm{s}$. The YIG cross has the following dimensions: the length of the each waveguide is $3.65 \mathrm{~mm}$; the width is $650 \mu \mathrm{m}$; and the YIG film thickness is $3.8 \mu \mathrm{m}$. Taking into account that the heat diffusion length at the above conditions is about $0.7 \mu \mathrm{m}$, less than $2 \%$ of the cross junction area has been exposed to high temperature. The latter explains the negligible effect of the laser ablation procedure on the saturation magnetization of the cross structure, which is $4 \pi M_{0}=1750 \mathrm{Oe}$. There are four $\Pi$-shaped micro-antennas fabricated on the edges of the cross. Antennas were fabricated from a gold wire of thickness $24.5 \mu \mathrm{m}$ and placed directly at the top of the YIG surface. The antennas are connected to a Keysight N5221A Programmable Network Analyzer (PNA) via a splitter, phase shifters, and attenuators. The splitter SigaTek SP542 is capable of operating at frequencies up to $18 \mathrm{GHz}$ and has an insertion loss of $1.7 \mathrm{~dB}$. The phase shifters are ARRA 9428A. The phase shifters provide up to $360^{\circ}$ degree phase rotation at frequency up to $8 \mathrm{GHz}$ with an internal insertion loss of $0.5 \mathrm{~dB}$. The step attenuators AC9011-90-11provide an adjustable attenuation up to $10 \mathrm{~dB}$ with $1 \mathrm{~dB}$ steps. The insertion loss of the attenuators is $0.5 \mathrm{~dB}$.

The testbed is placed inside GMW Systems Electromagnet, which generates a uniform bias magnetic field directed in-plane with the cross surface and along the virtual line connecting antennas \#1 and \#3 as shown in Fig. 2(A). The bias field is required for control of the spin wave transport. Prior to the experiments, we found a combination of the operational frequency $f=3.442 \mathrm{GHz}$ and the bias magnetic field $\mathrm{H}=627 \mathrm{Oe}$, which shows prominent propagation for the BVMSW and MSSW types of waves. The wavelength of the spin wave propagating from the input antennas to the output antenna is not constant within the cross structure. Besides, the wave number $k$ is a subject of modification in the center of the cross due to the change of the waveguide geometry. The maximum range of the wave numbers can be estimated as follows [15]:

$$
\Delta k \leq 2 \pi \Delta f / v_{g} \text {. }
$$

Taking $\Delta f=120 \mathrm{MHz}$ and $v_{g}=3 \times 10^{6} \mathrm{~cm} / \mathrm{s}$, the maximum wave number $k$ is around $120 \mathrm{~cm}^{-1}$, which correspond to the wavelength of $500 \mu \mathrm{m}$.

We started our experiments with the imaging of the YIG testbed without any object on top. The data collected in Figure $3(\mathrm{~A})$ show a holographic image of the testbed itself. Red markers in Fig.3(A) correspond to the inductive voltage measured at port 2. The total input power injected from PNA before the splitter is $-15 \mathrm{dBm}$. The maximum inductive voltage corresponding to the constructive spin wave interference is $3.7 \mathrm{mV}$. The minimum voltage corresponding to the destructive spin wave interference is $0.03 \mathrm{mV}$. The computer generated surface in Fig.3(A) is aimed to visualize the position of output maxima and minima. The results of numerical simulations are shown in Fig.3(B). There is a good agreement 
between the experimental data and the results of numerical modeling based on the simplified model Eqs. (3-6) showing the same position of maxima and minima. The absolute difference between the measured and calculated inductive voltage does not exceed $0.25 \mathrm{mV}$. This agreement is mainly due to the structure symmetry. In this case, the output voltage is maximized when both phases of each injected spin wave is equal and minimized when there is approximately a $\pi$ phase shift between the two interfering waves as evident from the experimental results.

Next, we obtained a holographic image of a piece of MFM tip made from highly doped, single crystal silicon, with $40 \mathrm{~nm}$ Co coating (product of Nanoworld company). The scanning electron microscopy (SEM) image of the sample is shown in Figure 4(A). The sample appears as trapezoidal shape. It has the following dimensions: $524.2 \mu \mathrm{m} \times 283.4 \mu \mathrm{m}$. The thickness of the sample is approximately $1 \mathrm{~mm}$. Magnetic properties of the sample were measured using a Vibrating Sample Magnetometer (VSM) (Lakeshore 7400 Series) at room temperature. Hysteresis loop measurements using field values of up to 1.6 $\mathrm{T}$ were obtained in order to plot mass normalized magnetization, $\sigma$ [emu/g] vs. applied field, $\mathrm{H}$ [Oe]. Coercivity, $H_{c}[\mathrm{Oe}]$, remanence magnetization $M_{r}[\mathrm{emu} / \mathrm{g}]$ and saturation magnetization $M_{s}[\mathrm{emu} / \mathrm{g}]$ were extracted from the $\sigma$ vs. $\mathrm{H}$ hysteresis curves. Saturation magnetization normalized magnetization $M / M_{s}$ vs. applied field, $\mathrm{H}[\mathrm{Oe}]$ is plotted in Fig.4(B). It shows saturation magnetization of $\sim 2500 \mathrm{Oe}$ and coercivity of $\sim 101$ Oe for the MFM tip object. The holographic image of the object is shown in Fig. 4(C). There are maxima around $3.5 \mathrm{mV}$ of the inductive voltage. The absolute minimum voltage corresponding to the destructive spin wave interference is $0.394 \mathrm{mV}$. The obtained image mainly resembles the one obtained for the testbed structure in Fig.3(A). This fact can be explained by taking into account relatively weak magnetic field produced by the sample compare to the external magnetic field of $627 \mathrm{Oe}$.

In Figure 5, we present data obtained for the $\mathrm{YIG}$ cross with a $\mathrm{SrFe}_{12} \mathrm{O}_{19}$ sample. The SEM image of the sample is shown in Figure $5(\mathrm{~A})$. The dimensions of the sample are: $778.3 \mu \mathrm{m} \times 426.2 \mu \mathrm{m} \times 1 \mathrm{~mm}$. $\mathrm{SrFe}_{12} \mathrm{O}_{19}$ is a hard permanent magnet. The results of magnetic characterization using VSM are shown in Fig.5(B). It shows saturation magnetization about $10 \mathrm{kOe}$ and coercivity of $3.6 \mathrm{kOe}$. The holographic image of the sample is presented in Fig. 5(C). The image appears different from the ones obtained for the testbed or testbed with Si/Co sample. There are multiple maxima and minima of non-equal height and depth, which differs drastically from the images presented in Fig.3(A) and Fig.4(C). The absolute maximum of inductive voltage is $1.2 \mathrm{mV}$. There are multiple local maxima of the inductive voltage between $1.05 \mathrm{mV}$ to $1.13 \mathrm{mV}$. The absolute minimum voltage corresponding to the destructive spin wave interference is $0.066 \mathrm{mV}$. Local minima of the inductive voltage appear at the level less than $0.5 \mathrm{mV}$. The maximum voltages are detected at symmetric phase combinations: $(0.6 \pi, 1.8 \pi)$ and $(1.8 \pi, 0.6 \pi)$. At the same time, one can observe certain asymmetry in the image (e.g. the position of minima). This sophisticated inductive voltage landscape reflects the strong and non-uniform magnetic field produced by the sample. 
In order to validate our hypothesis on the origin of multiple maxima and minima due to the effect of local magnetic field on spin wave propagation, we carried out a control experiment with non-magnetic conducting sample. The SEM image of the copper sample is shown in Figure 6(A). The sample appeared also as trapezoidal shape. The dimensions of the sample are $573.0 \mu \mathrm{m} \times 322.3 \mu \mathrm{m}$. The thickness of the sample is approximately $1 \mathrm{~mm}$. The results of magnetic characterization using VSM are shown in Fig.6(B). The sample shows diamagnetic response with neither saturation magnetization no coercivity. The holographic image of the sample is shown in Fig. 6(C). The shape of the image resembles the one of the testbed as shown in Fig.3, while with smaller amplitude. For instance, the maximum inductive voltage obtained for the copper sample is only $3.0 \mathrm{mV}$ compare to the maximum of $3.7 \mathrm{mV}$ obtained for the testbed. This fact is very well explained by taking into account an additional losses introduced to spin wave transport by the presence of the conducting material [15]. Thus, the presence of a non-magnetic conducting material does not change the shape of the holographic image but reduces the level of the output inductive voltage.

To complement experimental data obtained for objects of different materials, we present the results of numerical modeling. The numerical modeling has been performed assuming a three terminal cross bar geometry where the spin wave signals are injected from port 1 and port 3 and output is measured at port 2. The injected spin wave signals in our numerical model propagate through the first and third arms of crossbars and interfere at the center of the crossbar, where the specific sample under consideration is placed, producing the output signal. The injected spin waves at port 1 and port 3 have an amplitude 2 $\mathrm{meV}$. The amplitude change and the phase shift of the propagating spin wave $\sigma_{\mathrm{ij}}$ and $\Delta_{\mathrm{ij}}$ are extracted from the experimental data. Additional phase shift and damping is provided to the propagating input spin wave at the center of the cross depending on what kind of magnetized sample is placed at the center. However, since the sample size under consideration is too big for numerical micromagnetic simulation, we assumed a uniform contribution to each mesh from the sample. Each sample under consideration provides a unique phase and amplitude change which is evident from the unique output signal. Fig.7(A-C) shows simulated holographic images obtained for the low-coercivity Si/Co sample, high-coercivity $\mathrm{SrFe}_{12} \mathrm{O}_{19}$ sample and diamagnetic copper samples as presented in Fig.4(C), Fig.5(C), and Fig.6(C), respectively. For MFM tip showed in Fig.7(A), the phase difference and damping for both incoming spin waves at the center of the cross is close to zero. For the simulated image of the $\mathrm{SrFe}_{12} \mathrm{O}_{19}$ sample as in Fig $7(B)$, the damping is close to zero but phase difference between the two-incoming wave is close to $180^{\circ}$. For copper sample the situation is identical for MFM tip, but the diamagnetism of copper provides additional damping which is taken as $-4 \mathrm{~dB}$ in our simulations There is a good agreement in the cases of $\mathrm{Si} / \mathrm{Co}$ and diamagnetic samples, which mainly resembles the image of the testbed. In the case of the diamagnetic copper sample, the sample interacts with the propagating surface spin wave weakly. It provides lower amplitude output due to the damping. However, since the diamagnet provided identical 
phase shift to each spin wave coming from port 1 and port 3 , the phase of the output spin wave remains almost same compared as to the case of the testbed itself. In the case of "strong" high-coercivity $\mathrm{SrFe}_{12} \mathrm{O}_{19}$ sample, the holographic image changes drastically compare to the previous examples. In this case, the phase shift of the spin waves coming from port 1 and port 3 would not be the same since if the hard axis of the strong magnet is pointing to the direction of spin wave propagation, the phase shift would be minimal and for the opposite case, the phase shift would be maximum. Since the spin wave injected from port 1 and port 3 would be propagating in opposite direction, for one wave there would be minimal phase shift and for another, phase shift due to strong magnet would be maximum, and at the output, there would be minimal amplitude where the phase of the two waves coincide from the injecting port. The results of numerical modeling resemble the general features of the output, though the simplified model based on Eqs. (3-6) may not exactly reproduce the positions of multiple maxima and minima.

Finally, we carried experiments imaging samples with different amount of $\mathrm{SrFe}_{12} \mathrm{O}_{19}$ powder. The optical and SEM images of the powder is shown in Fig.8(A) and Fig.8(B). The powder has been packed in glass pipets. We used three samples containing $0.3 \mathrm{mg}, 0.75 \mathrm{mg}$ and $1.5 \mathrm{mg}$ of powder as shown in Fig. $8(C)$. The relative thickness of the samples ranges from $0.30 \mathrm{~mm}$ to $2.8 \mathrm{~mm}$. The results of VSM measurements in Fig.8(D) show almost identical M-H loops obtained in the normalized units M/Ms for all three samples. It shows saturation magnetization above $10 \mathrm{kOe}$. The coercivity field ranges from 655 to 771 Oe. In Fig.9, we present obtained holographic images of the three samples complemented by the results of numerical modeling. Experimental data for $0.3 \mathrm{mg}$ sample are shown in Fig. 9(A). The maximum inductive voltage is $3.5 \mathrm{mV}$. This sample mainly resembles the results obtained for the testbed. This fact can be explained taking the small amount of magnetic powder. The image changes significantly for $0.75 \mathrm{mg}$ sample as shown in Fig.9(B). This difference can be explained by the asymmetry in the phase changes for the two interfering spin waves provided by the sample. The latter smashes the interference effect by lowering maxima and enhancing minima due to the dephasing. In Fig.9(C), we present the holographic image of the sample with $1.5 \mathrm{mg}$ of magnetic powder. As in case of $0.3 \mathrm{mg}$ sample, one can clearly see the maxima and minima corresponding to the constructive and destructive spin wave interference. However, the position of maxima and minima is inverted compare to the image in Fig.9(A). This change can be attributed to the asymmetry and a $\pi$-phase shift between the interfering waves produced by the sample. These arguments are well supported by the results of numerical modeling presented in Fig.9.

\section{Discussion}

The magnonic holographic imaging technique described in this work is based on the spin wave interaction with magnetic objects where magnetic field produced by the object affects the interference pattern produced by the two propagating spin waves. The idea of holographic imaging is inspired by the 
recent advances of spin wave spectroscopy - a technique primarily used for spin wave study in a variety of magnetic structures including thin films [21] and nano-dots [22]. There are two key steps in the described magnonic holographic imaging technique: (i) the obtaining of an image of the magnetic object via its interaction with spin waves, and (ii) the extracting of information on the object magnetic structure by the obtained image. The data presented in our work demonstrate the feasibility of obtaining spin wave images of magnetic structures. As one can see from the obtained results, different magnetic structures can be visualized and classified by their interaction with spin waves. The images differ in the level of the transmitted signal and the position of maxima and minima. This difference is related to the unique data magnetic field profile produced by the object of interest. The results of numerical modeling obtained by the simplified model allows us to capture only the general difference between the samples, while a more elaborated model is required for a qualitative analysis. The imaging technique itself can be evolved in several ways by (i) increasing the number of input and output ports; (ii) modifying the geometry of the testbed; (iii) placing the object of interest inside the testbed for increasing the coupling with propagating spin waves; (iv) repeating imaging on several operational frequencies; (v) applying the time-domain measurements. The reconstruction of the magnetic field profile of the object by the holographic image is a direction for further research.

It is important to choose a proper set of parameters such as the operational frequency $f$, bias magnetic field $H$, and the input power $P_{i n}$, to minimize the non-linear magnon processes, which may disrupt the information form the object of interest. In general, the 3-magnon scattering processes are possible under the following condition

$$
f>f_{H} \text {, }
$$

where of $f_{H}=\gamma H$. Our experiments were accomplished at the bias magnetic field $\mathrm{H}=627 \mathrm{Oe}$. Taking into account $y=2.8 \mathrm{MHz} / \mathrm{Oe}$, the 3-magnon cut off frequency is about $3.5 \mathrm{GHz}$, which is higher than the operational frequency. The three-magnon processes are forbidden in this case. At the same time, the input microwave power did not exceed $-18 \mathrm{dBm}(15 \mu \mathrm{W})$. The level of the input power was chosen at least $10 \mathrm{~dB}$ below than the four-magnon parametric instability threshold for the studied structure. Typically only $10 \%$ of the input power is converted into the spin waves. Thus, the maximum spin wave power $P_{\text {msw }}$ in YIG film does not exceed $P_{\text {msw }} \leq 1.5 \mu \mathrm{W}$. The power can be related to the spin wave amplitude as follows:

$\mathrm{P}_{\mathrm{msw}}=|m|^{2} v d w$,

where $v$ is the group velocity, $d=3.8 \mu \mathrm{m}$ is YIG film thickness, $w=650 \mu \mathrm{m}$ is YIG film width, $|\mathrm{m}|$ is the spin wave amplitude. In turn, the amplitude is related to the angle of the magnetization precession cone $\varphi$ :

$|m|=\varphi \cdot M_{S}$.

Eqs.(8-10) allow us to estimate the maximum angle of the magnetization precession:

$\varphi=\sqrt{\frac{P_{m s w}}{M_{S}^{2} v d w}}$. 
Taking the group velocity $v=4 \times 10^{6} \mathrm{~cm} / \mathrm{s}$, the maximum angle of precession value $\varphi_{\max } \leq 0.0016$.

This value is much lower than the threshold value $\varphi_{t h}$ of the 4-magnon parametric instability which can be estimated as follows [15]:

$\varphi_{t h} \approx \sqrt{\frac{\Delta \mathrm{H}}{4 \pi M}}$.

Taking $\Delta H=0.5 O e$, we obtain $\varphi_{t h}=0.018$, which is much lower than the estimate for the maximum angle of magnetization precession by Eq.(11): $\varphi_{\max }<\varphi_{t h}$.

It should be noted that Eq. (8) is valid for an infinite film. A more rigorous analysis would require the consideration of the effect of the shape anisotropy. The experimental data presented in the previous Section show no feasible sign of the non-linear effects in the chosen range of parameters.

Noise is composed of undesirable signal components that arise from various sources in the electronic or, like in our case, magnonic imaging system [23]. The electronic noise in detector microantennas and inevitable testbed structure imperfections are the major physical factors limiting the sensitivity and space resolution of the magnonic imaging. There are different physical processes resulting in the fluctuation of the output inductive voltage including electronic noise in the input antennas, magnetization fluctuation in the testbed and the object of interest, or fluctuations in the bias magnetic field. Using the recording of the output signal in the time domain we calculated the electrical current noise spectral density, $S_{1}$, as a function of frequency by applying the fast Fourier transform. The extracted noise spectral density in the low-frequency limit was in the range from $10^{-12} \mathrm{~A}^{2} / \mathrm{Hz}$ to $10^{-8} \mathrm{~A}^{2} / \mathrm{Hz}$. This noise level is comparable with conventional deep sub-micrometer semiconductor technologies [24]. The noise immunity of the proposed magnonic imaging system can be partially attributed to the low flicker noise level in ferrite structures, which typically does not exceed $-130 \mathrm{dBm}$ [25]. One should also note that spin waves are not sensitive to the structure imperfections with dimensions much shorter than the operational wavelength. A good agreement between the experimental and theoretical data obtained in this work is in part due to the micrometer-scale operation wavelength. Downscaling the testbed along with the operational wavelength may result in modifications of the noise spectral density [24-25]. The effect of scaling on the noise in magnetic structures is mainly unexplored and reserved for future study.

High space resolution is the major potential advantage of the spin wave spectroscopy compare to other techniques such as ferromagnetic resonance (FMR). Similar to the optical microcopy, the space resolution of the spin wave imaging (i.e. the minimum feature size of the magnetic field profile produced by the magnetic object) is limited by the spin wave wavelength. However the wavelength of magnons is orders of magnitude shorter than that of electromagnetic waves (photons) of the same frequency, which provides the possibility of nanometer scale resolution at $\mathrm{GHz}$ operation frequency. There are several important advantages of using two or several spin wave sources for magnetic object imaging compare to the techniques using just one excitation source [21]. The use of interference makes it possible to enhance the sensitivity and locality of the spin wave measurements. The change in the interference pattern occurs 
mainly due to the dephasing among the interfering spin waves caused by the presence of the magnetic object rather than the change of the spin wave amplitude (e.g. experiments with magnetic powder shown in Fig.9). The ability to control the phase difference between the two spin wave generating antennas is another important advantage inherent in magnonic holographic imaging. At some point, the changing of the phase difference between the input spin waves is equivalent to the rotating the angle of object illumination. In optics, the angle of illumination is accomplished mechanically by literally rotating the light source. In the case of spin waves, the use of the phased array of antennas makes it possible to create an input spin wave front of an arbitrary form without any physical movement. This advantage has been recently utilized for spin wave-based pattern recognition [17].

The need in the external bias magnetic field is one of significant disadvantages associated with the use of a micrometer scale testbed. It makes impossible to recognize tiny signatures of "weak" magnetic objects with coercivity is less than the bias field. In this case, the holographic image mainly resembles the one of the testbed. This problem can be resolved by utilizing testbeds made of antiferromagnetic materials, which do not require a bias magnetic field for spin wave propagation. The excitation of high-frequency short-wavelength spin waves is another technical challenge. The use of micro-antennas has certain practical limits related to the size of the antenna. Micro-focus BLS technique [26], multifferoic elements [27], and spin torque-oscillators [28] are among the most promising approaches towards nanometer scale spin wave excitation and detection.

Finally, we want to outline the unique feature of magnonic holographic imaging, which is the ability of sensing magnetic objects located inside a magnetic matrix. FMR technique also provides such capability of resolving magnetic composites by the difference in their frequency resonance but with very limited space resolution. The use of micro- and nanometer scale spin waves would preserve all the advantages of FMR technique and complement it with ultimate space resolution. Potentially, magnonic holographic imaging may complement the existing imaging techniques and be utilized for non-destructive in-situ magnetic object characterization.

\section{Conclusions}

In this work, we have described a technique for magnetic microstructures imaging via their interaction with propagating spin waves. The proposed technique allows us to visualize magnetic structures as $3 \mathrm{D}$ objects in a phase-inductive voltage space. We presented several holographic images obtained of different magnetic objects. The shape of the objects reflects the effect of magnetic field produced by the object on the propagating spin waves. In our experiments, we used a cross junction made of $\mathrm{Y}_{3} \mathrm{Fe}_{2}\left(\mathrm{FeO}_{4}\right)_{3}$ with four micro-antennas fabricated on the arms of the cross. All experiments are done at room temperature. Experimental data is complemented by the results of numerical modeling. 
These first results are aimed to demonstrate the appealing properties of the magnonic holographic approach.

\section{Acknowledgement}

The authors thank Prof. J. Shi for access to Vibrating Sample Magnetometer. This work was supported by the Spins and Heat in Nanoscale Electronic Systems (SHINES), an Energy Frontier Research Center

funded by the U.S. Department of Energy, Office of Science, Basic Energy Sciences (BES) under Award \# SC0012670. 


\section{Figure Captions}

Figure 1. (A) Illustration of the magnonic holographic imaging. The object of interest is placed on top of the testbed made of material with low spin wave damping. The testbed has a cross shape with four microantenna fabricated at the edges of the cross arms. Two spin waves are excited by the antennas (e.g. \#1 and \#3) connected to the same RF source. The phase of the input spin wave signals (e.g. Phase 1 and Phase 2) are independently controlled by the phase shifters. One antenna (e.g. \#2) is used for the detection of the inductive voltage produced by the spin wave interference. The interference pattern depends on input phase difference as well as the accumulated phase shift caused by the magnetic field produced by the object. (B) Example of magnonic holographic image. It is a $3 \mathrm{D}$ plot, where the output voltage is plotted as a function of the two the input spin wave phases. The position of maxima and minima correspond to the constructive and destructive spin wave interference, respectively. The image reflects the unique signature of the object via tis interaction with propagating spin waves.

Figure 2. (A) Experimental setup schematics. The testbed is a cross junction made of single crystal yttrium iron garnet $\mathrm{Y}_{3} \mathrm{Fe}_{2}\left(\mathrm{FeO}_{4}\right)_{3}$ The length of the each arm is $3.65 \mathrm{~mm}$; the width is $650 \mu \mathrm{m}$; and the YIG film thickness is $3.8 \mu \mathrm{m}$. There are four $\Pi$-shaped micro-antennas fabricated on the edges of the cross. The antennas are connected to a Keysight N5221A Programmable Network Analyzer (VNA) via a splitter (depicted as S), attenuators (depicted as A), and phase shifters (depicted as Ph). Two antennas located at port \#1 and \#3 are connected to the output of PNA providing RF signal of power $-15 \mathrm{dBm}$. Antenna located at port \#2 is used for the detection of inductive voltage produced by the spin wave interference and connected to the input of PNA. (B) The testbed is placed inside a GMW Electromagnet, which provides a uniform bias magnetic field directed in-plane with the cross surface and along the virtual line connecting antennas \#1 and \#3. (C) Photo of the device packaged with SMA cables connected to the four ports.

Figure 3. (A) Holographic image of the testbed with no object on top. The red markers depict the measured inductive voltage. The computer generated surface connecting the markers is aimed to visualize the position of maxima and minima corresponding to constructive and destructive spin wave interference. (B) Results of numerical modeling showing the image of the YIG cross.

Figure 4. (A) SEM image of a piece of MFM tip made from highly silicon with $40 \mathrm{~nm}$ Co coating. (B) Results of VSM measurements showing M-H loop of the object. (C) Holographic image of the object.

Figure 5. (A) SEM image of the $\mathrm{SrFe}_{12} \mathrm{O}_{19}$ sample. (B) Results of VSM measurements showing M-H loop of the object. (C) Holographic image of the object.

Figure 6. (6) SEM image of the copper sample. (B) Results of VSM measurements showing diamagnetic properties of the object. (C) Holographic image of the object. 
Figure 7. Results of numerical modeling based on the Eqs. (3-6). (A) Simulated image of the MHM tip as in Fig. 4(C). (B) Simulated image of the $\mathrm{SrFe}_{12} \mathrm{O}_{19}$ sample as in Fig. $5(\mathrm{C})$. (C) Simulated image of the copper sample as in Fig. 6(C).

Figure 8. (A) Optical image of the $\mathrm{SrFe}_{12} \mathrm{O}_{19}$ powder sample. (B) SEM image of the $\mathrm{SrFe}_{12} \mathrm{O}_{19}$ powder sample. (C) Results of VSM measurements showing $\mathrm{M}-\mathrm{H}$ loop of the samples with $\mathrm{SrFe}_{12} \mathrm{O}_{19}$ powder. The curves show the normalized magnetization $\mathrm{M} / \mathrm{Ms}$ of the samples containing $0.3 \mathrm{mg}, 0.75 \mathrm{mg}$, and $1.5 \mathrm{mg}$ of $\mathrm{SrFe}_{12} \mathrm{O}_{19}$ powder. (D)Photo of the $\mathrm{SrFe}_{12} \mathrm{O}_{19}$ powder samples placed on the top of the testbed.

Figure 9. Experimental data and the results of numerical modeling showing the holographic images of the $\mathrm{SrFe}_{12} \mathrm{O}_{19}$ powder samples. (A) Sample with $0.3 \mathrm{mg}$ of $\mathrm{SrFe}_{12} \mathrm{O}_{19}$ powder. (B) Sample with $0.75 \mathrm{mg}$ of $\mathrm{SrFe}_{12} \mathrm{O}_{19}$ powder. (C) Sample with $1.5 \mathrm{mg}$ of $\mathrm{SrFe}_{12} \mathrm{O}_{19}$ powder. 


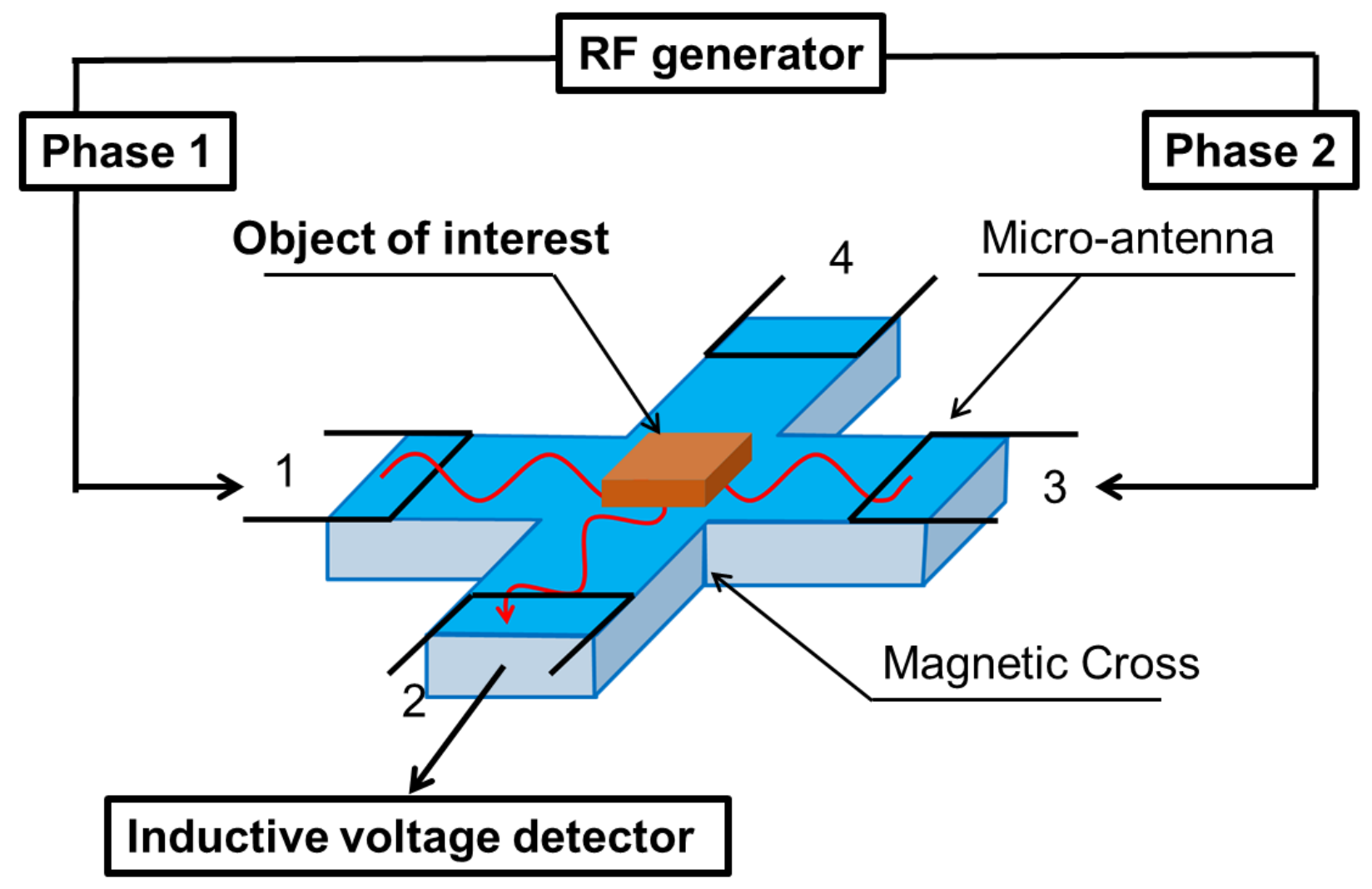

Figure 1 

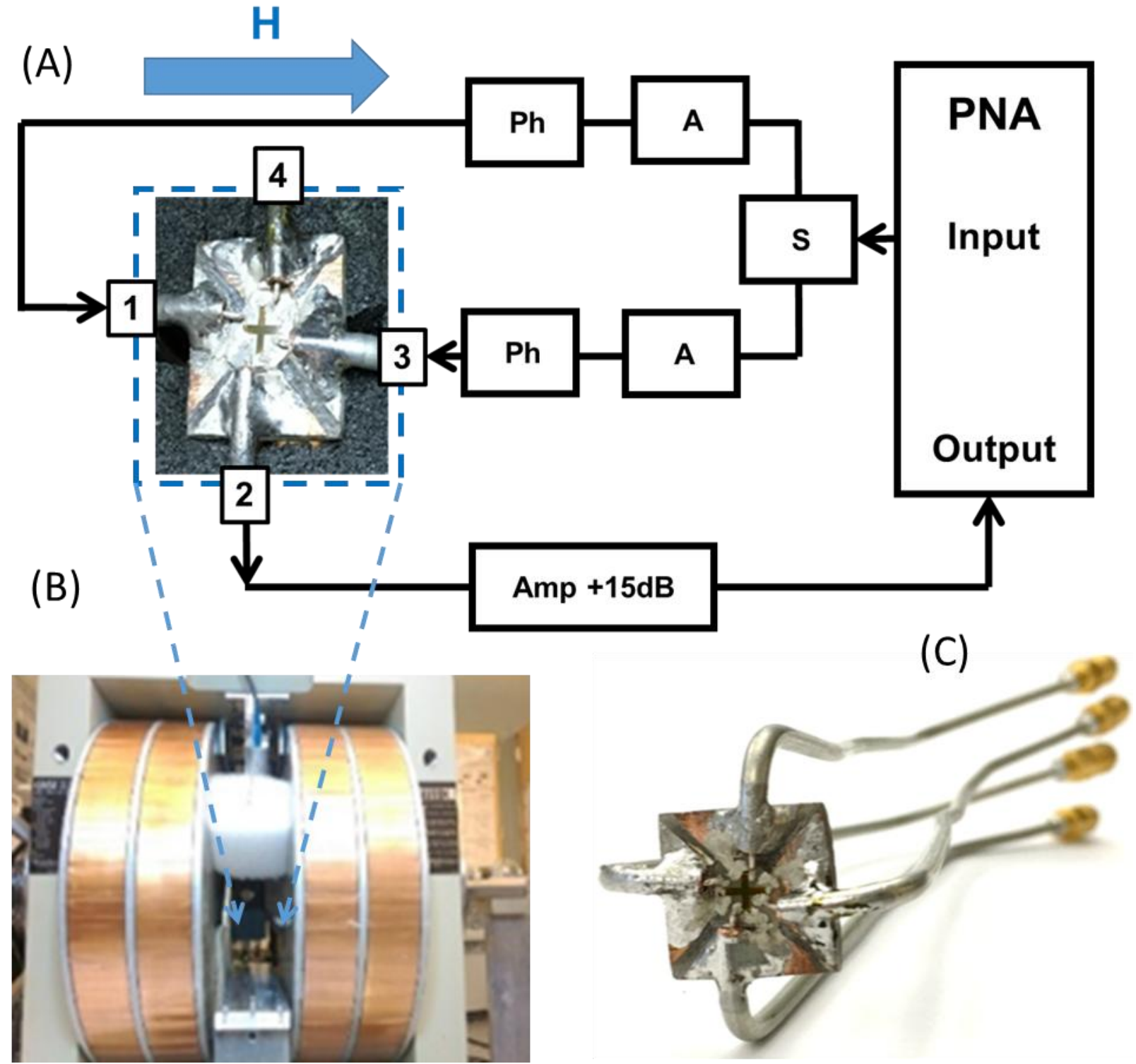

Figure 2 
(A)

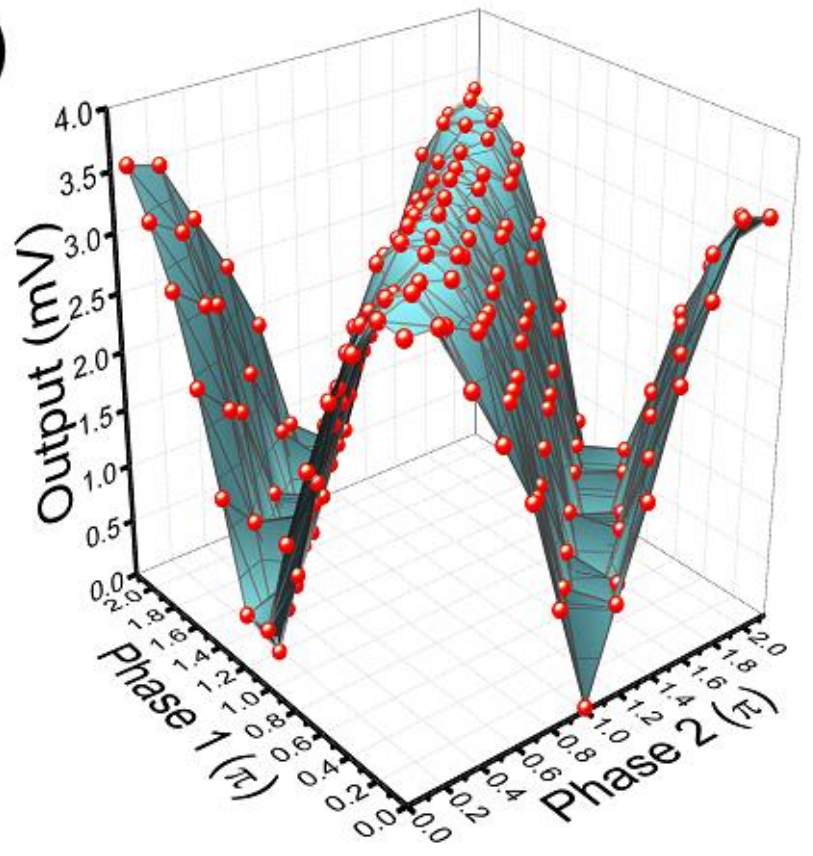

(B)

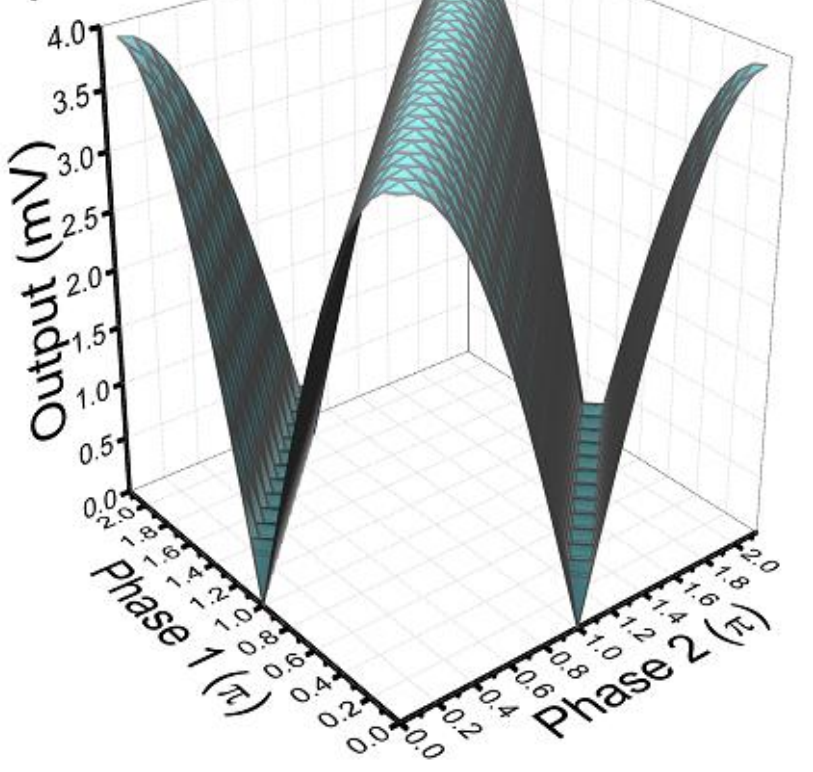

Figure 3 
(A)
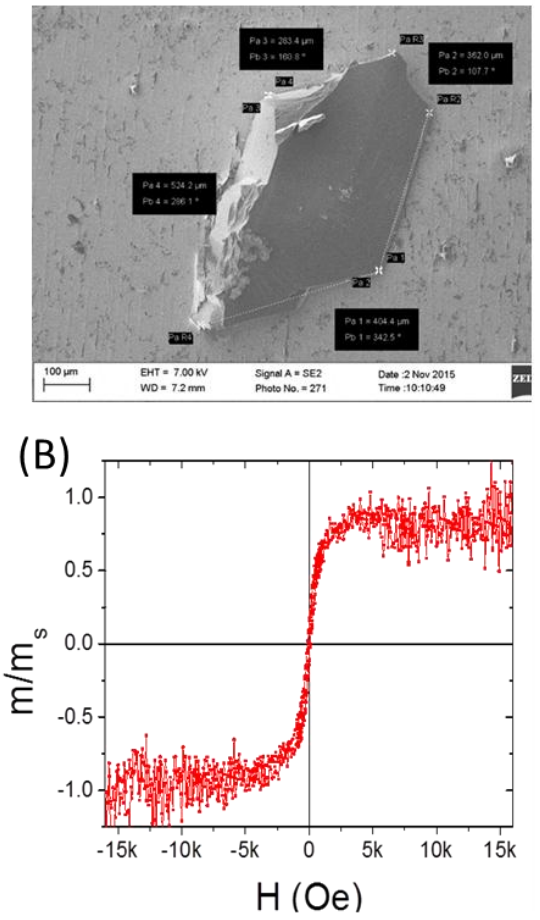

(C)

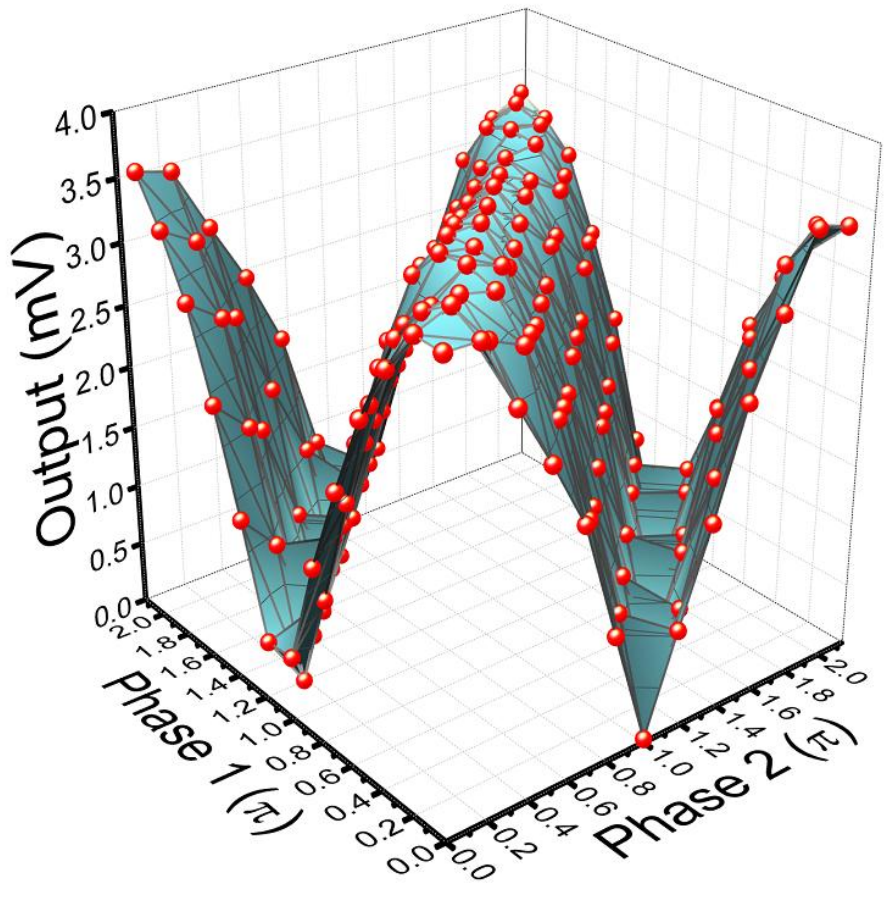

Figure 4 
(A)
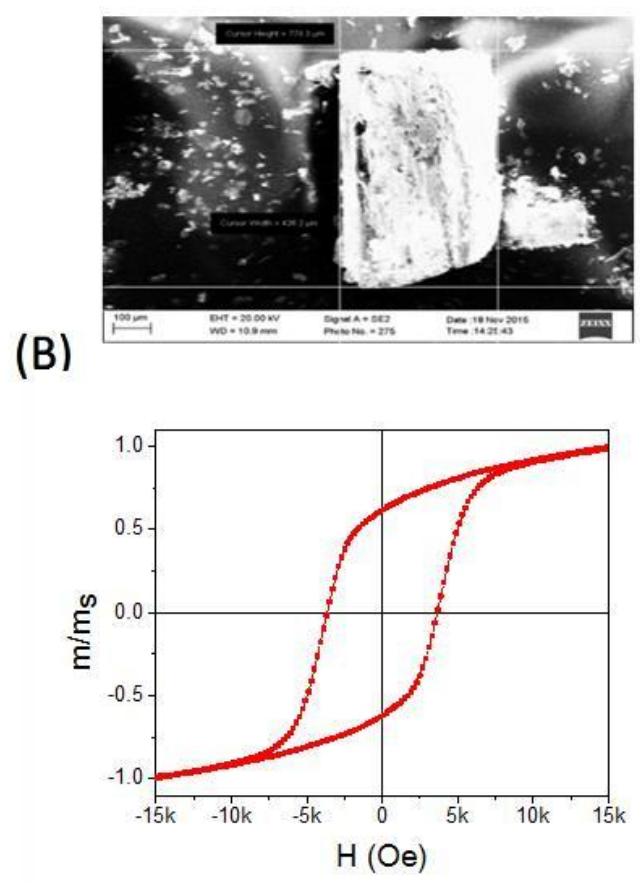

(C)

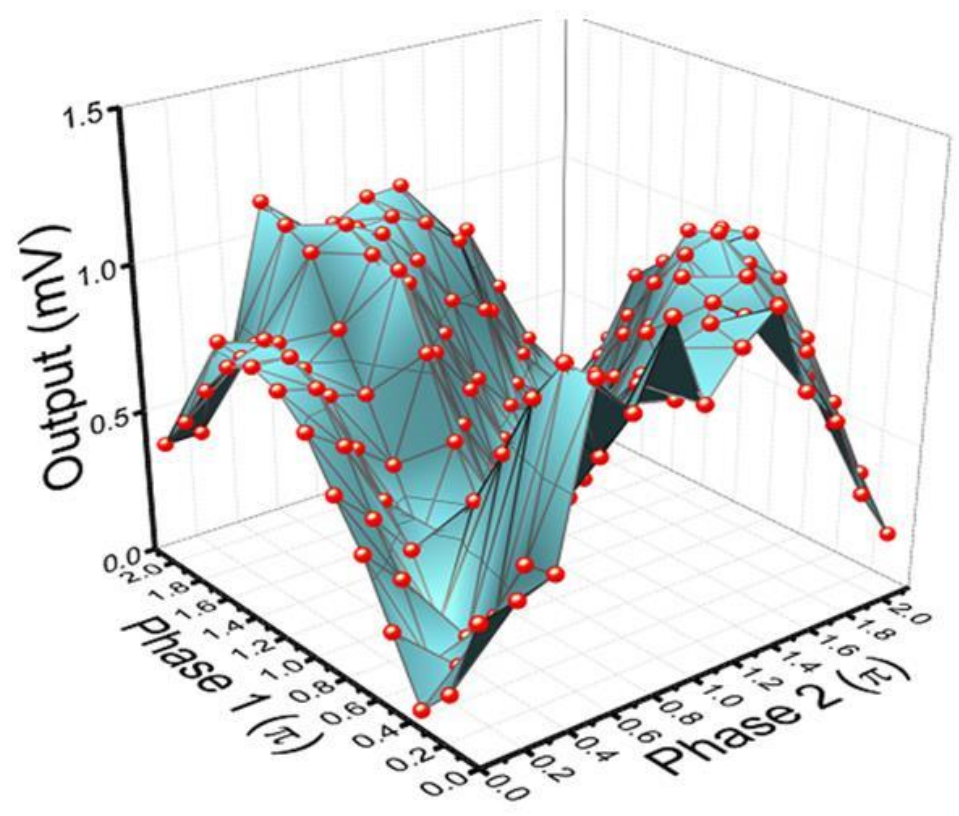

Figure 5 

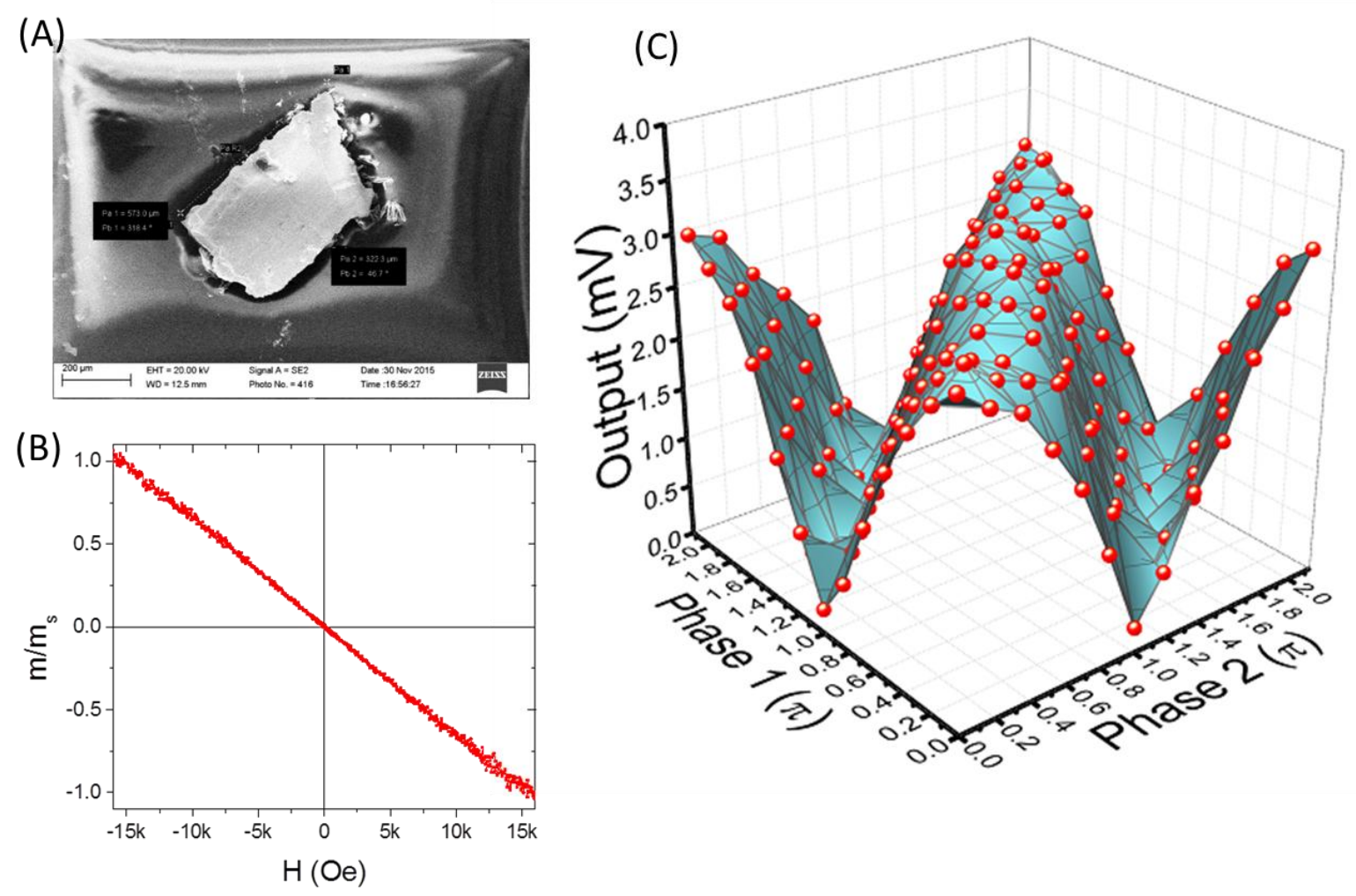

Figure 6 


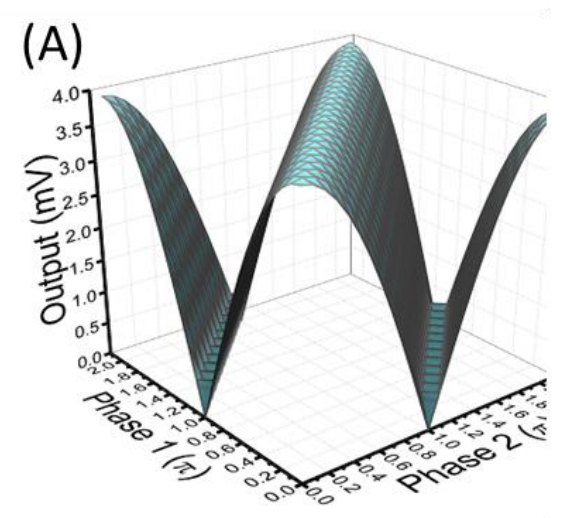

(B)

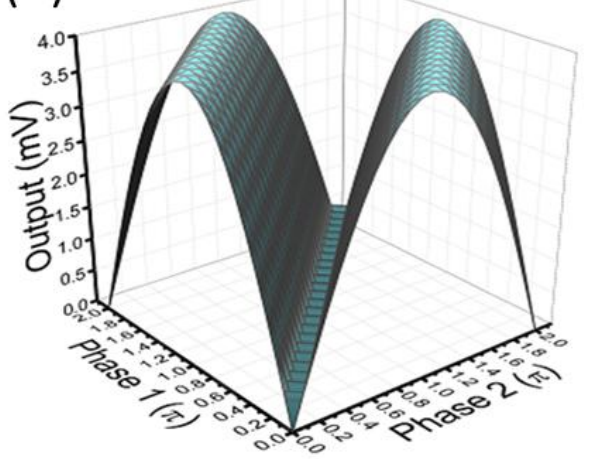

(C)

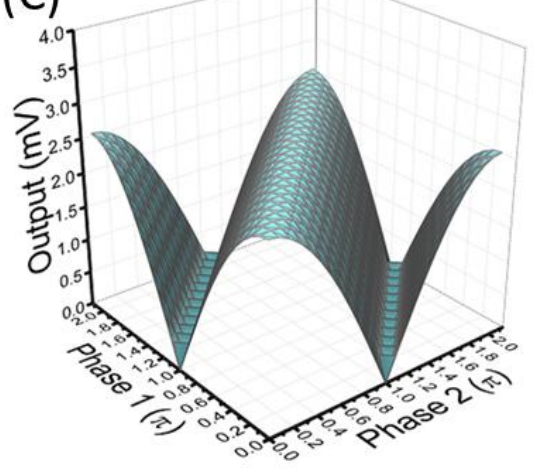

Figure 7 


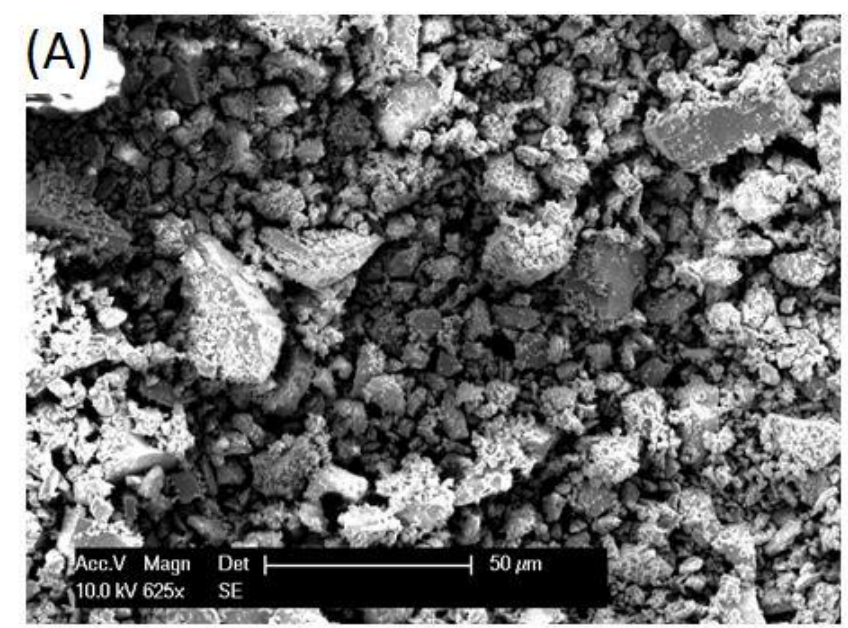

(C)

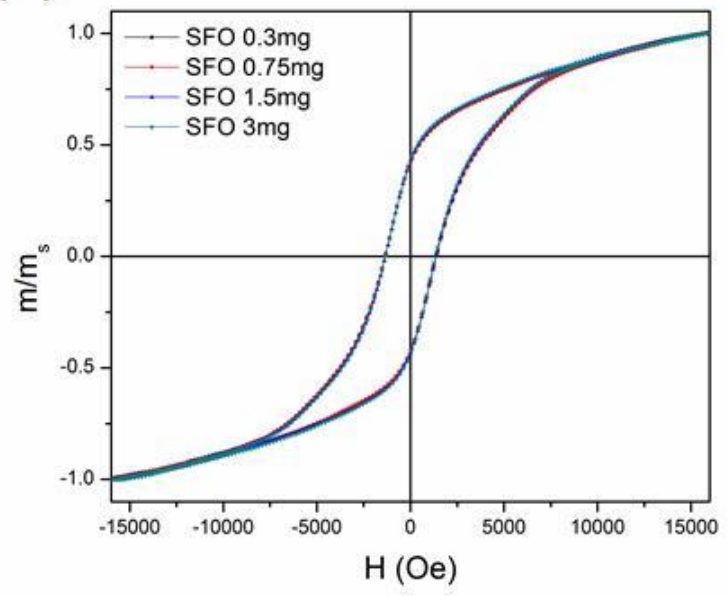

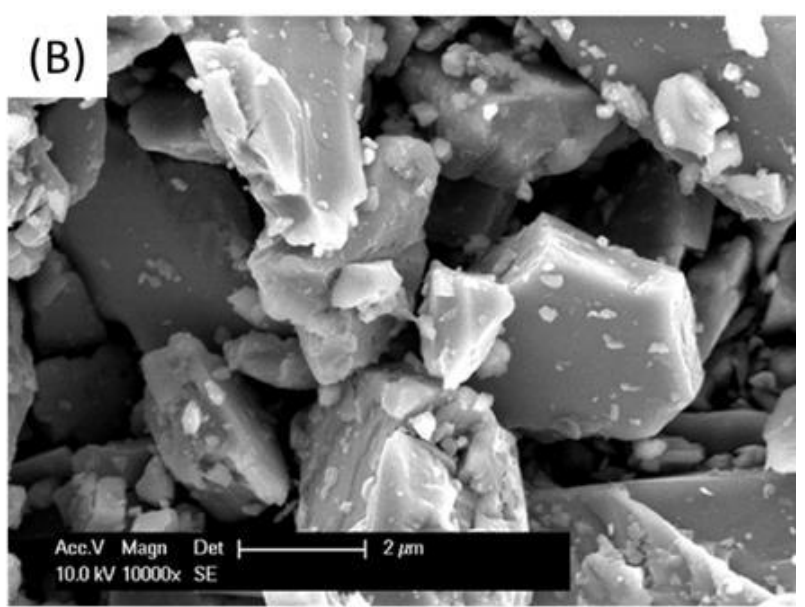

(D)

$0.3 \mathrm{mg} \quad 0.75 \mathrm{mg} \quad 1.5 \mathrm{mg}$

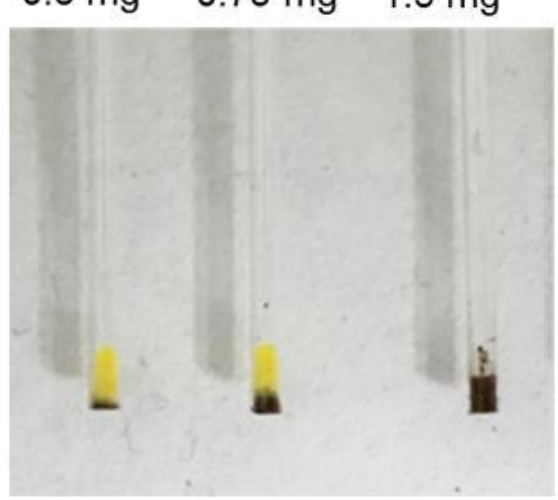

Figure 8 


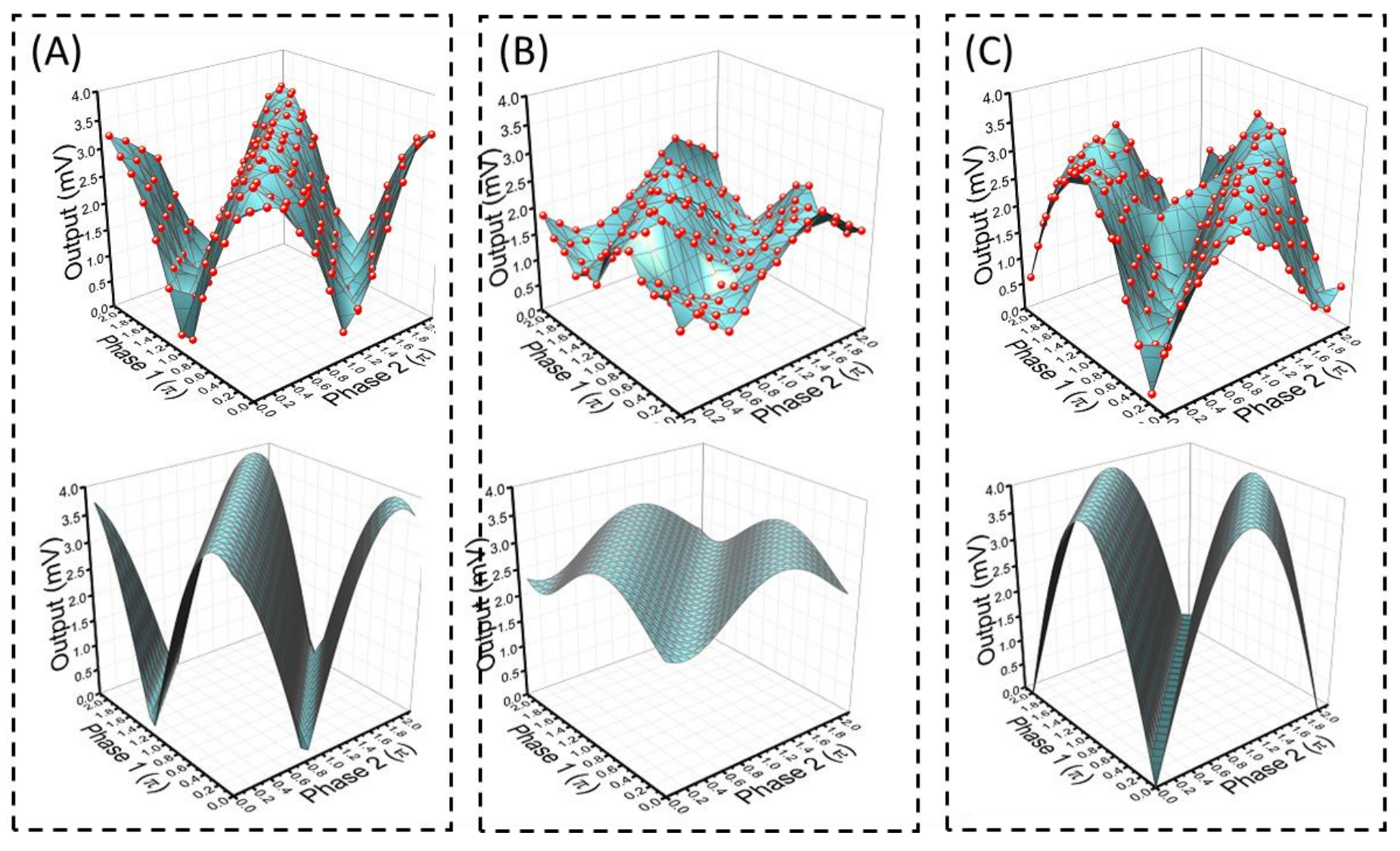

Figure 9 


\section{References}

1. Terris, B.D. and T. Thomson, Nanofabricated and self-assembled magnetic structures as data storage media. Journal of Physics D-Applied Physics, 2005. 38(12): p. R199-R222.

2. Yadollahpour, A., Magnetic Nanoparticles in Medicine: A Review of Synthesis Methods and Important Characteristics. Oriental Journal of Chemistry, 2015. 31: p. 271-277.

3. Baker, K.F., A review of magnetic bubble memories and their applications. Radio and Electronic Engineer, 1981. 51(3): p. 105-16.

4. Tumanski, S., Modern magnetic field sensors - a review. Przeglad Elektrotechniczny, 2013. 89(10): p. 1-12.

5. Kim, T. and T. Hyeon, Applications of inorganic nanoparticles as therapeutic agents. Nanotechnology, 2014. 25(1).

6. Rugar, D., et al., MAGNETIC FORCE MICROSCOPY - GENERAL-PRINCIPLES AND APPLICATION TO LONGITUDINAL RECORDING MEDIA. JOURNAL OF APPLIED PHYSICS, 1990. 68(3): p. 1169-1183.

7. Weinberger, P., John Kerr and his effects found in 1877 and 1878. Philosophical Magazine Letters, 2008. 88(12): p. 897-907.

8. Fischer, P., X-Ray Imaging of Magnetic Structures. IEEE Transactions on Magnetics, 2015. 51(2): p. 1-31.

9. Chapman, J.N., High resolution imaging of magnetic structures in the transmission electron microscope. Materials Science and Engineering: B, 1989. 3(4): p. 355-358.

10. Angeloni, L., et al., Experimental issues in magnetic force microscopy of nanoparticles, in Nanoforum 2014, M. Rossi, et al., Editors. 2015.

11. Huebner, J. and M. Oestreich, Time-Resolved Spin Dynamics and Spin Noise Spectroscopy, in Spin Physics in Semiconductors, M.I. Dyakonov, Editor. 2008. p. 115-134.

12. Jersch, J., et al., Mapping of localized spin-wave excitations by near-field Brillouin light scattering. Applied Physics Letters, 2010. 97(15).

13. Serga, A.A., A.V. Chumak, and B. Hillebrands, YIG MAgnonics. Journal of Physics D: Applied Physics, 2010. 43(26).

14. Kostylev, M.P., et al., Spin-wave logical gates. Applied Physics Letters, 2005. 87(15): p. 1535011-3.

15. Gurevich, A.G. and G.A. Melkov, eds. Magnetization Oscillations and Waves. 1996, CRC Press Inc., New York.

16. Gertz, F., et al., Magnonic Holographic Memory. Magnetics, IEEE Transactions on, 2015. 51(4): p. 4002905-400910.

17. Kozhevnikov, A., et al., Pattern recognition with magnonic holographic memory device. Applied Physics Letters, 2015. 106(14).

18. Landau, L.D. and E.M. Lifshitz, Theory of the dispersion of magnetic permeability in ferromagnetic bodies. Phys. Z. Sowietunion, 1935. 8: p. 153.

19. Gilbert, T.L., Physical Review, 1955. 100: p. 1243.

20. Silva, T.J., et al., Inductive measurement of ultrafast magnetization dynamics in thin-film Permalloy. JOURNAL OF APPLIED PHYSICS, 1999. 85(11): p. 7849-7862.

21. Covington, M., T.M. Crawford, and G.J. Parker, Time-resolved measurement of propagating spin waves in ferromagnetic thin films (vol 89, art no 237202, 2002). Physical Review Letters, 2004. 92(8).

22. Bailleul, M., R. Hollinger, and C. Fermon, Microwave spectrum of square permalloy dots: quasisaturated state. Physical Review B (Condensed Matter and Materials Physics), 2006. 73(10): p. 104424-1-14. 
23. Balandin, A.A., ed. Noise and Fluctuation Control in Electronic Devices 2002, American Scientific Publisher: Los Angeles.

24. Claeys, C., A. Mercha, and E. Simoen, Low-frequency noise assessment for deep submicrometer CMOS technology nodes. Journal of the Electrochemical Society, 2004. 151(5): p. G307-G318.

25. Rubiola, E., Y. Gruson, and V. Giordano, On the flicker noise of ferrite circulators for ultra-stable oscillators. leee Transactions on Ultrasonics Ferroelectrics and Frequency Control, 2004. 51(8): p. 957-963.

26. Demidov, V.E., et al., Excitation of short-wavelength spin waves in magnonic waveguides. Applied Physics Letters, 2011. 99(8).

27. Cherepov, S., et al., Electric-field-induced spin wave generation using multiferroic magnetoelectric cells. Applied Physics Letters, 2014. 104(8): p. 082403 (5 pp.)-082403 (5 pp.).

28. Demidov, V.E., S. Urazhdin, and S.O. Demokritov, Direct observation and mapping of spin waves emitted by spin-torque nano-oscillators. Nature materials, 2010. 9(12): p. 984-988. 